International Journal of Engineering \& Technology, $7(4.38)(2018) 91-95$
International Journal of Engineering \& Technology
SPC
Website: www.sciencepubco.com/index.php/IJET
Research paper

\title{
Development of Optimization Model of Budget Allocation for Promotion of Unmanned Aerial Vehicles
}

\author{
Anna Anatolievna Burdina ${ }^{1 *}$, Marina Nikolaevna Kaloshina ${ }^{1}$, Elena Timofeevna Manaenkova ${ }^{1}$, Anna Alexandrovna \\ Nehrest $^{1}$, Tatyana Mikhailovna Rogulenko ${ }^{1}$
}

${ }^{1}$ Moscow Aviation Institute (State University of AerospaceTechnologies), Volokalamskoe Highway, 4, Moscow, 125993, Russia

*Corresponding author E-mail: burdina.a.a@bk.ru

\begin{abstract}
This article discusses manufacturers of unmanned aerial vehicles, convex and stochastic programming, quantile optimization in economy are reviewed. Problem of budget allocation for promotion of engineering products is defined and solved in two variants: deterministic formulation, when a company is new and has not its own statistic data, and, otherwise, stochastic formulation. These problems were solved using methods of optimization theory and theory of stochastic systems such as simplex method, successive quadratic programming, and confidence method. Optimization model of budget allocation for promotion of unmanned aerial vehicles has been developed which optimizes budget for innovative engineering products.
\end{abstract}

Key words: budget optimization, unmanned aerial vehicles, quadrocopters, SEO, convex programming, stochastic programming, quantile optimization.

\section{Introduction}

Engineering industry is one of the leading and largest industries of Russian economy. It is responsible for $1 / 5$ of total production and $1 / 3$ of all employees in national economy. The share of engineering in structure of Russian industry is $19 \%$. At present Russian engineering is comprised of 19 large complex industries and more than 100 subindustries and productions. The industry includes metallurgical engineering, railway car manufacture, diesel engine manufacture, boiler manufacture, turbine manufacture, atomic engineering, printing machine production, as well as metal processing and robotics $[1,2]$.

Engineering products play decisive role in implementation of achievements of R\&D progress in all industries. More than $30 \%$ of total scope of industrial products are manufactured by engineering complex, however, in terms of this performance Russia is behind such countries as Japan, Germany, and the USA where engineering share in total industrial structure is $40-50 \%$. Leading industries of precise engineering are instrumentation, radio-technic and electronic engineering, electrical engineering. An actively developed sector of engineering and robotics is manufacture of unmanned aerial vehicles (UAV, also known as drones) - aircrafts without a human pilot aboard [3, 4]. UAV can possess various degree of independence: from remote control to complete automation, also they can be characterized by design, purpose and other numerous parameters. UAV can be controlled by episodic commands or continuously, in the latter case UAV are referred to as remotely piloted aerial vehicle (RPAV) [4]. The main advantage of UAV/RPAV is significantly lower cost of their manufacture and operation (under equal efficiency of preset tasks) - according to expert appraisals the cost of combat UAV of higher complexity level is about USD 6 million whereas the cost of comparable piloted fighter aircraft is about USD 100 million [5]. A disadvantage of UAV is vulnerability of remote control which is especially important for military UAV $[6,7]$.

According to Russian Regulations of Airspace Utilization, UAV is defined as an aircraft conducting a flight without pilot (crew) aboard and controlled automatically, by operator from control post, or by combination of the mentioned methods [8]. The United States Department of Defense applies similar definition where the only sign of UAV is the absence of a pilot [9]. International Civil Aviation Organization (ICAO) subdivides radio-controlled models and UAV, pointing out that the first are intended mainly for entertainment and should be governed by local and not international regulations of airspace utilization [1]. The number of quadrocopter manufacturers increases every year aiming both at civil and at military demands. The list below summarizes manufacturers of civil quadrocopters $[10,11]$.

The major quadrocopter manufacturers are as follows:

- 3D Robotics (3DR) - US company manufacturing UAV both for professionals and for amateurs. The most popular and marketable quadrocopter model is 3DR IRIS+.

- Aeryon Systems - a company which in addition to personal quadrocopters manufactures military, commercial and social security UAV. The flag quadrocopters are SkyRanger and Scout.

- Align - a company established in Taiwan in 1984. Manufacturer of various devices and gadgets, from 2013 also manufactures Align quadrocopters. The most well-known one is Align 480L.

- DJI Innovations (commonly known as DJI) - the most wellknown global manufacturer of quadrocopters for amateurs and professionals. The headquarter is located in Shenzhen (China). Their Phantom 2 Vision+ is one of the best quadrocopters with camera $[12,13]$.

- Draganfly - a Canadian company established in 1998 which manufactures high-quality quadrocopters with onboard camera. Draganflyer X6 model is a particular proud of the company.

- Hubsan - another well-known company for quadrocopter enthusiasts. The company is located in China, the most wellknown models are SpyHawk and X4.

- Nine Eagles - a Chinese company established in 2008 in Shanghai. It manufactures radio-controlled models including 4- 
rotor quadrocopters. Galaxy Visitor series is the most popular model.

- Parrot - a leading quadrocopter manufacturer, the most wellknown model is Parrot AR.Drone 2.0. The company was established in France in 1994, in addition to drones it manufacturers other useful gadgets.

- WLToys - the trade mark of Shantou Chenghai Welei Toys Industrial Co. located in China. Wide range of radio-controlled models is provided including quadrocopters, the most well-known model is V959.

In Russia quadrocopters are manufactured by ZALA Aero Group, Izhevsk (ZALA 421-02X, ZALA 421-06), as well as by OOO Aviarobots, OOO Autonomous Aerospace Systems-GeoService) (Dan-2) [14].

Occurrence of new technologies in the markets cardinally changed promotional practices. At present businessmen can use such promotional practices as website promotion or social media marketing. Internet all over the World is considered to be the best trading platform for various products. Modern web advertising has nearly unlimited capabilities to promote any product. The most popular practices are contextual and banner advertisings as well as website promotion using SEO [6,7].

Nowadays SEO is an independent business activity. Therefore, it is required to develop a model for promotion of UAV (quadrocopters) and software for optimization of cash distribution among advertising media [10].

\section{Methods}

Let us formulate the problem of budget optimization for UAV (quadrocopters) with regard to $N$ key phrases: requests. Demand for each request for current month $S_{i}$ depends on position in search results. Each position has its cost $c_{i}$ [10]. Hence, demand for each request depends on the amount which should be allocated for promotion of the $i$-th request $S_{i}\left(c_{i}\right)$. Herewith, only a portion of Internet users effect a purchase. There exists coefficient of conversion equaling to the ratio of visitors having effected purchase to total number of visitors who followed the $i-$ th link These are independent random variables $k_{i}$, with normal distribution $N\left(\tilde{k}_{i}, \sigma_{i}\right)$. In order to calculate profit, it is required to know marginality $m_{i}-$ average profit on order. Upon optimization it is necessary to account for demand which should be met by seller for each request $Q_{i}$ and allocated budget $C$. In addition, it is necessary to consider for other expenses $c_{0}$. [1, 15] Let us introduce the following notations:

$C$ - budget allocated for advertisement;

$c_{i}$ - money required for promotion of the $i$-th request;

$c_{0}$ - other expenses;

$S_{i}\left(c_{i}\right)$ - demand for the $i$-th request;

$Q_{i}$ - demand which can be met by seller for the $i$-th request;

$m_{i}-$ average profit on the $i-$ th order;

$k_{i} \sim N\left(\tilde{k}_{i}, \sigma_{i}\right)-$ coefficients of conversion;

$N-$ amount of promoted requests, where $i=1 . . N$.

Let us define the profit function $f(\bar{c})$ and use it as the target function of optimization problem. On one $i-$ th request we receive profit equaling to product of demand, coefficient of conversion and marginality: $k_{i} \cdot S_{i}\left(c_{i}\right) \cdot m_{i}$. It also necessary to subtract $c_{i}$ money which should be used for promotion of the $i$-th request and other expenses [15].

Summing over $i$ from 1 to $N$, we obtain the following optimization problem with $2 N+1$ inequality constraints.

Problem 1.1:

$$
\left\{\begin{array}{c}
\sum_{i=1}^{N} k_{i} \cdot S_{i}\left(c_{i}\right) \cdot m_{i}-\sum_{i=0}^{N} c_{i} \rightarrow \max _{\mathrm{c}} \\
\sum_{i=0}^{N} c_{i} \leq C \\
S_{i}\left(c_{i}\right) \cdot k_{i} \leq Q_{i} \\
c_{i} \geq 0
\end{array}\right.
$$

Therefore, using realization of random value $k_{i}$, we obtained optimization problem in deterministic formulation.

In general case, random values $k_{i}$ are included into the target function and constraints. Hence, we have the problem with probabilistic constraints and probabilistic criterion. In practice two cases are possible: when the seller can completely meet the requested demand or when demand exceeds the seller capabilities. Thus, the profit function depending on this demand will be as follows:

$f(\bar{c})=\sum_{i=1}^{N} \min \left(k_{i} \cdot S_{i}\left(c_{i}\right), Q_{i}\right) \cdot m_{i}-\sum_{i=0}^{N} c_{i}$

Since $k_{i}$ has normal distribution and is included linearly into the profit function, then $f(\bar{c})$ is also a random variable with normal distribution. Let us the quantile function for $f(\bar{c})$ :

$f(\bar{c})_{\alpha}=\min \left\{\varphi: P_{\varphi}(f(\bar{c})) \geq \alpha\right\}, \alpha \in(0,1)$

where $P_{\varphi}(f(\bar{c}))=P\{f(\bar{c}) \geq \varphi\}$ is the probability function for the profit function.

Therefore, we obtain the following problem of quantile optimization [12]:

Problem 1.2:

$$
\left\{\begin{array}{c}
\left(\sum_{i=1}^{N} \min \left(k_{i} \cdot S_{i}\left(c_{i}\right), Q_{i}\right) \cdot m_{i}-\sum_{i=0}^{N} c_{i}\right)_{\alpha} \rightarrow \min _{\mathrm{c}} \\
\sum_{i=0}^{N} c_{i} \leq C \\
c_{i} \geq 0
\end{array}\right.
$$

Analysis of solution methods of linear programming problems. Graphical method is used for solution of linear programming problems with two variables: $x_{1}, x_{2}$. In order to apply this method, it is required to plot a set of permissible solutions $X$ on plane $x_{1}, x_{2}$ determined by the constraints $g_{j}(x) \leq 0$.

On the same plane the level line of criterion function $f(x)=0$ is plotted and the vector $\nabla f(x)$ is calculated: the gradient of function showing its fastest growth. Then the boundary point of the set $X$ is determined where the target function is maximum or minimum.

The Danzig's simplex method is sued for any number of variables. The strategy of this method is based on the features of problem formulation. The set of permissible solutions $X$, defined by constraints $g_{j}(x) \leq 0$, is a convex set in the form of convex polytope with finite number of terminal points. The simplex method enumerates terminal points of the set $X$ until the basic solution is obtained [12].

Solution methods of convex programming problems.

Let the convex and smooth function $f(x)$ is given. Smoothness is considered as continuity of its first order partial derivatives.

Definition. The function $f(x): R^{m} \rightarrow R$ is convex at the set $X \subseteq$ $R^{m}$, if $\forall \lambda \in[0,1]$ and $\forall x_{1}, x_{2} \in X$, the following is valid:

$f\left(\lambda x_{1}+(1-\lambda) x_{2}\right) \leq \lambda f\left(x_{1}\right)+(1-\lambda) f\left(x_{2}\right)$

Let the convex closed set of permissible solutions $X$ is given defined as follows: 
$g_{j}(x) \leq 0, j=1 . . p$

where $g_{j}(x)$ is the convex functions. Let us formulate the following problem of convex programming:

$$
\left\{\begin{array}{c}
f(x) \rightarrow \min _{X} \\
g_{j}(x) \leq 0, j=1 . . p \\
x_{i} \geq 0
\end{array}\right.
$$

Statement. In equations (1.1)-(1.3) the local minimum coincides with the global one.

Definition. Epigraph of function $f(x): R^{m} \rightarrow R$ is the set $\{(u, \varphi): f(u) \leq \varphi\}$.

Convexity criterion: the function $f(x): R^{m} \rightarrow R$ is convex if and only if its epigraph is a convex set.

Let us consider several solution methods of convex programming [16].

Linear approximation method at each iteration applies linear approximation of the target function $f(x)$ and the constrain functions $g_{j}(x)$ in the vicinity of current point $x^{k}$ :

$f^{k}(x)=f\left(x^{k}\right)+\left(\nabla f\left(x^{k}\right),\left(x-x^{k}\right)\right)$

$g_{j}^{k}(x)=g_{j}\left(x^{k}\right)+\left(\nabla g_{j}\left(x^{k}\right),\left(x-x^{k}\right)\right)$

Instead of convex problem, auxiliary problem of linear programming is solved:

$\left\{\begin{array}{c}\min _{x^{k} \in X^{k}} f^{k}(x)=f^{k}\left(x^{k+1}\right) \\ X^{k}=\left\{x: g_{j}^{k}(x) \leq 0\right\}\end{array}\right.$

In such description the point $x^{k+1}$ can be beyond the range of permissible values. In order to eliminate such situation, the following solution is obtained:

$x^{k+1}=x^{k}+\lambda^{k}\left(\tilde{x}^{k}-x^{k}\right)$

where $\tilde{x}^{k}$ is the solution of equation (1.5) and the step $\lambda^{k}$ is determined by one of the following methods:

1) $\quad \lambda^{k}$ is determined as a solution of one-dimensional optimization problem $f\left(x^{k}+\lambda^{k}\left(\tilde{x}^{k}-x^{k}\right)\right) \rightarrow \min$ at $\lambda^{k} \in[0,1]$.

2) Assuming that $\lambda^{k}=1$, according to equation (1.5)

3) we calculate $x^{k+1}$ and the value of the target function

$f\left(x^{k+1}\right)$. If $f\left(x^{k+1}\right)<f\left(x^{k}\right)$ is not met, then we randomly decrease $\lambda^{k}$ (for instance, two-fold). We reduce this step until the mentioned condition is met.

Successive quadratic programming method

SQP is one of the most advanced methods in nonlinear programming. This method is realized in three main stages [5]:

- Correction of Hessian matrix for Lagrange function:

$L(x, \lambda)=f(x)+\sum_{i=1}^{n} \lambda_{i} g_{i}(x)$

At each main iteration the Hessian matrix for Lagrange function is approximated as follows:

$H_{k+1}=H_{k}+\frac{q_{k} q_{k}^{T}}{q_{k}^{T} s_{k}}-\frac{H_{k}^{T} H_{k}}{s_{k}^{T} H_{k} s_{k}}$

where

$s_{k}=x_{k+1}-x_{k}$ $q_{k}=\nabla f\left(x_{k+1}\right)+\sum_{i=1}^{n} \lambda_{i} \nabla g_{i}\left(x_{k+1}\right)-\left(\nabla f\left(x_{k}\right)+\sum_{i=1}^{n} \lambda_{i} \nabla g_{i}\left(x_{k}\right)\right)$

- Solution of quadratic programming problem.

At each iteration of SQP the QP problem is solved in order to determine the further search direction $d$ :

$\underset{d \in R^{n}}{\operatorname{minimize}} q(d)=\frac{1}{2} d^{T} H d+c^{T} d$

$A_{i} d=b_{i}, \quad i=1 . . m$

$A_{i} d \leq b_{i}, \quad i=m+1 . . n$

The solution is comprised of two stages. The first stage is calculation of the most probable point. The second stage is generation of an iterative sequence of the most probable points which is already converged to the required solution $[6,14]$.

- Calculation of linear search and return function

Solution of QP subproblem gives formation of vector $d$, which in its turn is used for calculation of the following point:

$x_{k+1}=x_{k}+\alpha_{k} d$

In order to obtain sufficient decrease in the return function the length parameter of step $\alpha_{k}$ is evaluated. The return function realized in this algorithm is as follows [7, 12]:

$\Psi(x)=f(x)+\sum_{i=1}^{m} r_{i} g_{i}(x)+\sum_{i=m+1}^{n} r_{i} \max \left(0, g_{i}(x)\right)$

Herewith, the penalty parameter is:

$r_{i}=\left(r_{k+1}\right)_{i}=\max _{i}\left\{\lambda_{i}, \frac{1}{2}\left(\left(r_{k}\right)_{i}+\lambda_{i}\right)\right\}, \quad i=1 . . n$

In the adopted method the penalty parameter as initial parameter is as follows:

$r_{i}=\frac{\|\nabla f(x)\|}{\left\|\nabla g_{i}(x)\right\|}$

Such approach provides significant contribution of constraints with moderate parameters into penalty parameters, which is particularly important for active constraints near the solution point.

Least square method

The most popular approximation method of experimental data is the least square method. This method makes it possible to use approximating functions of random type and is referred to the group of global methods.

Proximity criterion in the least square method is requirement of minimum sum of squared deviations from approximating function to approximated point:

$\sum_{i=1}^{n}\left(y_{i}-f\left(x_{i}\right)\right)^{2} \rightarrow \min$

Therefore, it is not required that approximating function passes all preset points, which is particularly important upon approximation of data with obvious errors $[17,18]$.

A peculiar feature of the method is that the approximating function can be random. Its type is determined by peculiarities of problem to be solved, for instance, by physical considerations when results of physical experiment are approximated. The most frequently applied are straight line approximation (linear regression), approximation by polynomials (polynomial regression), approximation by linear combination of random functions. 
Solution problems of stochastic programming problems.

Confidence method

Let us consider a family of confidence set:

$\mathcal{F}_{\alpha}=\left\{S: S \in \mathcal{B}\left(R^{N}\right), P(S) \geq \alpha\right\}, \alpha \in(0,1)$.

Let $S \in \mathcal{F}_{\alpha}$. Let us consider the maximum function:

$$
\psi(S, \bar{c})=\sup _{\bar{k} \in S} f(\bar{k}, \bar{c})
$$

and minimax problems:

$$
\overline{\mathrm{c}}^{S}=\arg \min _{\bar{c} \in X} \psi(S, \bar{c}), \quad \psi^{S}=\inf _{\bar{c} \in X} \psi(S, \bar{c})
$$

Statement: $\psi(S, u) \geq \varphi_{\alpha}(u)$, that is, the maximum function $\psi(S, u)$ is the upper limit of quantile function $\varphi_{\alpha}(u)$ [8].

Let us consider generalized minimax problem:

$$
\left(\tilde{S}_{\alpha}, \tilde{u}_{\alpha}\right)=\arg \min _{S \in \mathcal{F}_{\alpha}} \min _{u \in U} \psi(S, \bar{c})
$$

The generlaized minimax problem (1.6) is equivalent to minimization problem of quantile function when the following conditions are valid:

$$
\begin{array}{ll}
\text { a) } & \psi\left(\tilde{S}_{\alpha}, \tilde{u}_{\alpha}\right)=\varphi_{\alpha}\left(u_{\alpha}\right) ; \\
\text { b) } & \psi\left(\tilde{S}_{\alpha}, \tilde{u}_{\alpha}\right)=\varphi_{\alpha}\left(\tilde{u}_{\alpha}\right) ; \\
\text { c) } & \text { if } U_{\alpha} \text { is the set of optimum strategies in quantile }
\end{array}
$$
oprimization problem, then for any $u_{\alpha} \in U_{\alpha}$ there exists a set $S_{\alpha} \in$ $F_{\alpha}$, such that $\left(S_{\alpha}, u_{\alpha}\right)=\varphi_{\alpha}\left(u_{\alpha}\right)$.

Theorem: If $\in(0,1)$, and $U_{\alpha} \neq \varnothing$, then the generalized minimax problem (1.6) is equivalent to the minimization problem of quantile function $[8,19]$.

Problem in deterministic formulation

Let us consider equation 1.1. We replace random values $k_{i}$ with their monthly calculated realizations. Depending on the type of demand function $S_{i}\left(c_{i}\right)$, the formulated problem can be converted to a problem of linear or convex programming. In any of these cases the promotion $\operatorname{cost} c_{i}(n)$ as a function of required position $n$ is a threshold function (Fig. 1).

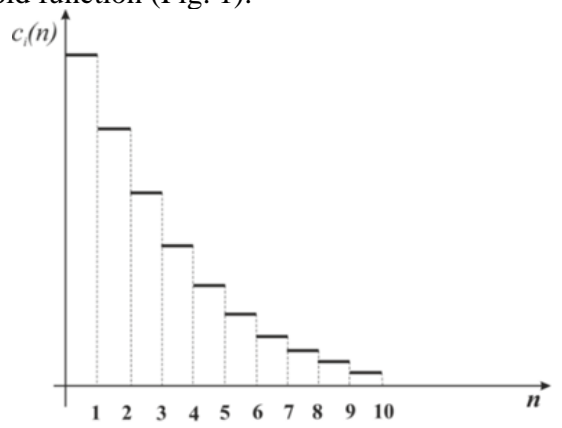

Fig. 1: Cost as a function of position in search results

Conversion to linear programming problem.

Let approximate $S_{i}\left(c_{i}\right)$ by linear function. Let $S\left(c_{i}\right)=a_{i} \cdot c_{i}+b_{i}$. Substituting $S\left(c_{i}\right)$ into equation 1.1, we obtain as follows:

$\sum_{i=1}^{N} k_{i}\left(a_{i} \cdot c_{i}+b_{i}\right) \cdot m_{i}-\sum_{i=1}^{N} c_{i} \rightarrow \max _{c}$

Then we transform the obtained criterion function:

$$
\sum_{i=1}^{N} k_{i}\left(a_{i} \cdot c_{i}+b_{i}\right) \cdot m_{i}-\sum_{i=1}^{N} c_{i}=\sum_{i=1}^{N}\left(k_{i}\left(a_{i} \cdot c_{i}+b_{i}\right) \cdot m_{i}-c_{i}\right)
$$

$$
\begin{gathered}
=\sum_{i=1}^{N}\left(k_{i}\left(a_{i} \cdot c_{i}+b_{i}\right) \cdot m_{i}-c_{i}\right)=\sum_{i=1}^{N}\left(k_{i}\left(a_{i} \cdot c_{i}+b_{i}\right) \cdot m_{i}-c_{i}\right) \\
= \\
=\sum_{i=1}^{N}\left(\left(k_{i} \cdot a_{i} \cdot m_{i}-1\right) \cdot c_{i}+k_{i} \cdot b_{i} \cdot m_{i}\right) \\
=\sum_{i=1}^{N} \tilde{a_{l}} \cdot c_{i}+\sum_{i=1}^{N} k_{i} \cdot b_{i} \cdot m_{i}
\end{gathered}
$$

where $\tilde{a_{l}}=k_{i} \cdot a_{i} \cdot m_{i}-1$.

Since $k_{i}, b_{i}, m_{i}$ are constants, the second sum can be rejected because it does not effect the coordinates of extreme point $[9,19]$.

$$
\left\{\begin{array}{c}
\sum_{i=1}^{N}{\widetilde{a_{i}}}_{i} \cdot c_{i} \rightarrow \max _{c} \\
\sum_{i=1}^{N} c_{i} \leq C \\
a_{i} \cdot c_{i}+b_{i} \leq Q_{i} \\
c_{i} \geq 0
\end{array}\right.
$$

\section{Results}

Let us consider the task of budget optimization for promotion of UAV (quadrocopters), provided that the request is displayed in the first page of search system, that is, in the first 10 lines, the so called Top-10, since according to Interlabs data, probability of opening the second page of search by the buyer is less than $5 \%$ [7].

Therefore, Problem 1.1 is converted to linear programming problem. Exactly from this file for further calculations we obtain request list [13], cost of inclusion into Top-10 for each request, marginality - average profit on order $m_{i}, S$ - constraint for maximum demand, and budget $c$ [18].

1) For each request in the list the software visits Yandex. Wordstat [5] and gains statistics on requests for the last month, thus determining demand in the formulated optimization problem.

2) Then the obtained data are sorted in descend order of demand, considering that the higher is the demand, the higher is the profit [5].

3) The software obtains data on conversions $\widetilde{k}_{l}$ using Yandex.Metrika [6].

4) The software forms output Excel template with equations for filling.

5) Then, the optimum solution is searched. All expenses on all requests are enumerated with decrease in profit and compared with constraints for budgets and demand. Herewith, profit is calculated. The enumeration is arranged so that when the software satisfies budget and constraints for demand, the optimization is terminated. Optimum values of variables will be stored in output file (Table 1). Table 1 summarizes optimization results: required expenses and optimum positions for higher profit. Budget for promotion of products is RUB124000.

Table 1: Budget optimization for promotion of UAV (quadrocopters)

\begin{tabular}{|c|c|c|c|c|c|c|}
\hline $\begin{array}{c}\text { Product of } \\
\text { engineering } \\
\text { industry }\end{array}$ & $\begin{array}{c}\text { De- } \\
\text { nand } \\
\text { unit }\end{array}$ & $\begin{array}{c}\text { Profit } \\
\text { per } \\
\text { unit, } \\
\text { (RUB) }\end{array}$ & $\begin{array}{c}\text { Expenses } \\
\text { for } \\
\text { ranking } \\
\text { as TOP } \\
10 \\
\text { (RUB) }\end{array}$ & $\begin{array}{c}\text { Conver } \\
\text { sion }\end{array}$ & $\begin{array}{c}\text { Position } \\
\text { (solution } \\
\text { of } \\
\text { optimiza } \\
\text { tion } \\
\text { problem) }\end{array}$ & $\begin{array}{c}\text { Expenses } \\
\text { to achieve } \\
\text { position } \\
\text { (RUB) }\end{array}$ \\
\hline $\begin{array}{c}\text { Quadrocopter } \\
1\end{array}$ & 546 & 30000 & 55000 & 0.015 & 7 & 78571.43 \\
\hline $\begin{array}{c}\text { Quadrocopter } \\
2\end{array}$ & 144 & 28000 & 45000 & 0.05 & 10 & 45000 \\
\hline & & & & & Total & $\mathbf{1 2 3 5 7 1 . 4 3}$ \\
\hline
\end{tabular}


In the course of studies the Python-based software was developed for optimization of budget distribution among advertising media for promotion of UAV (quadrocopters). Figures 2 and 3 illustrate schematically the block diagrams.

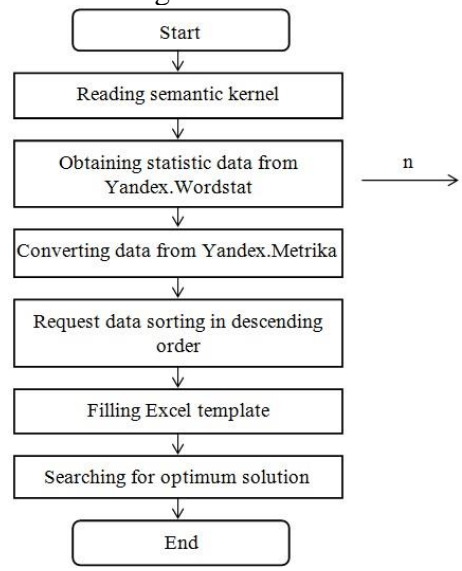

Fig 2: Schematic block diagram

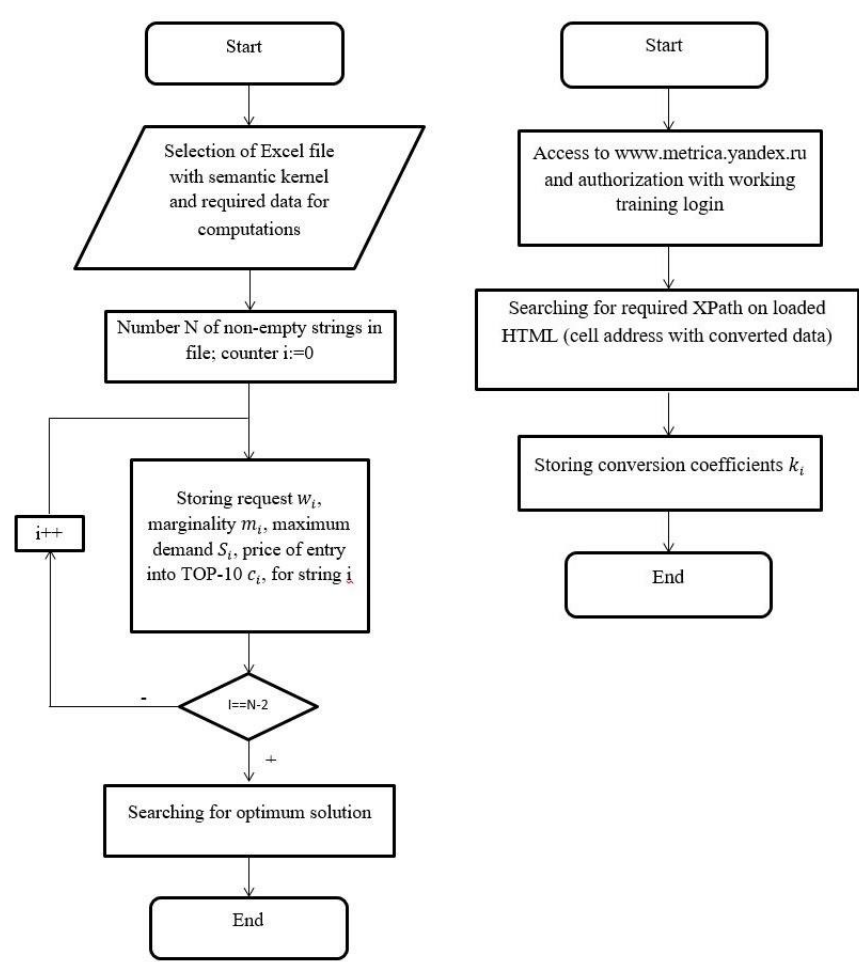

Fig. 3: Schematic block diagram of semantic kernel reading and obtaining data on conversions from Yandex.Metrika service

\section{Conclusion}

This article analyzed business activities of UAV manufactures. Necessity to promote quadrocopters as a commercial product of engineering industry was substantiated. Budget optimization model was developed for promotion of UAV (quadrocopters) as well as software for budgeting. The developed software loaded all required data from Internet and Excel file, thus expert activity on evaluation of advertisement costs was simplified and accelerated. Two formulations of the problems were considered: deterministic and stochastic. The obtained results coincided with the solutions obtained by optimization methods. Using the software, optimum budget allocation was determined for promotion of UAV (quadrocopters) up to positions 7 and 10.

\section{Acknowledgments}

We are grateful to our colleagues for their assistance and for financial support of the work.

\section{References}

[1] A.N. Troshin, A.A. Burdina, Formalizatsiya zadachi optimizatsii stoimosti korporativnykh struktur s uchetom faktora regional noi nadezhnosti [Formalization of cost optimization of corporative structures with accounting for regional reliability factor], Trudy MAI 49 (2011) 67-74.

[2] P. Flach, Machine Learning: The Art and Science of Algorithms That Make Sense of Data, Cambridge University Press, Cambridge, 2012.

[3] The most annoying in banners is intrusiveness. Superjob.ru research portal: https://www.superjob.ru/community/ad/60199/ Revised November 24, 2011, Accessed September 10, 2018

[4] SEOpromotion: http://ultra-com.net/seo-uslugi.

[5] Yandex.Wordstat https://wordstat.yandex.ru/ Accessed September 10, 2018.

[6] Yandex.Metrika https://metrika.yandex.ru Accessed September 10, 2018.

[7] Types of requests and clickability of positions in Yandex search. First three positions in Yandex search: http://www.seonews.ru/analytics/tipyi-zaprosov-i-klikabelnostpozitsiy-v-vyidache-yandeksa-3-mesta-v-vyidache-yandeksa/ Revised July 14, 2008, Accessed September 10, 2018.

[8] A.I. Kibzun, S.Yu. Kan, Zadachi stokhasticheskogo programmirovaniya $\mathrm{s}$ veroyatnostnymi kriteriyami [Stochastic programming problems with probability criteria], FIZMATLIT, Moscow, 2009.

[9] A.V. Degtyarev, V.A. Vdovin, A.M. Kovalev, aet al., Informatsionnye tekhnologii $\mathrm{v}$ menedzhmente [Information technologies in management], Guidebook, Dobroe slovo, Moscow, 2011.

[10] A.A. Burdina, Metodologicheskie aspekty prinyatiya upravlencheskikh reshenii $\mathrm{v}$ oblasti tsenoobrazovaniya $\mathrm{i}$ optimizatsii assortimenta produktsii [Methodological aspects of managerial solutions in the scope of cost formation and optimization of product assortment], Vestnik Moskovskogo Aviatsionnogo Instituta 1(13) (2006) 116-124.

[11] M. Bowles, Machine Learning in Python: Essential Techniques for Predictive Analysis, Wiley, Indianapolis, 2015.

[12] A.N. Troshin, A.A. Burdina, N.V. Moskvicheva, et al., Mechanism to analyze economic reliability of the innovational potential of aircraft enterprises, International Journal of Applied Business and Economic Research 14(14) (2016) 747-765

[13] W. Winston, Microsoft Excel Data Analysis and Business Modeling (5th Edition). Microsoft Press, 2017.

[14] H. Percival, Test-Driven Development with Python, 2nd Ed., O'Reilly Media, Sebastopol, 2017.

[15] E. Matthes, Python Crash Course: a hands-on, Project-Based Introduction to Programming, No Starch Press, San Francisco, 2016.

[16] A.A. Burdina, Vliyanie riskov na konkurentosposobnost predpriyatiya [Influence of risks on company competitiveness], Voprosy ekonomiki 2 (2006) 7.

[17] I. Ashmanov, A. Ivanov, Optimizatsiya i prodvizhenie saitov v poiskovykh sistemakh [Optimization and promotion of sites in searching systems], 3rd ed., Piter, St. Petersburg, 2011.

[18] A.V. Bodiako, S.V. Ponomareva, T.M. Rogulenko, et al., The goal setting of internal control in the system of project financing, International Journal of Applied Business and Economic Research 14(15) (2016) 11045-11063.

[19] T.R. Padmanabhan, Programming with Python, Springer, Singapore, 2016. 